\title{
La participación ciudadana en Chile. El caso de los Consejos Comunales de la Sociedad Civil en la región del Biobío
}

\section{Public participation in Chile.The case of the Community Councils of Civil Society in the Biobío region}

doi: http://dx.doi.org/10.32870/

espiral.v25i73.7006

\section{Resumen}

El presente artículo centra su atención en el proceso de implementación en Chile de la Ley 20.500, publicada el año 20II, sobre participación ciudadana en la gestión pública, $y$ en especial en uno de los mecanismos establecidos como obligatorio para las administraciones públicas locales: los Consejos Comunales de la Sociedad Civil ( $\mathrm{COSOC}$ ), en específico en los implementados en las municipalidades de la región del Biobío, en Chile. Se incluye la opinión de actores relevantes en esta materia respecto al proceso, así como se revisa la ley misma. El trabajo aporta al dejar en evidencia una inconsistencia entre el espíritu de la ley y las reales atribuciones de los COSOC.

Palabras claves: nuevo servicio público, participación ciudadana, Chile, control social, Consejos Comunales de la Socie-

dad Civil.

\author{
Waleska Tatiana Muñoz Aravena
}

\begin{abstract}
This article focuses on the implementation process of the Chilean law 20.500, ublished in $201 \mathrm{I}$, in relation with the citizen participation in public management and, in particular, on one of the mechanisms established as mandatory for local public administrations, such as the Community Councils of Civil Society (COSOCs), specifically on the councils of the Biobío region, in Chile. In addition, this article inquiries about the opinion of relevant actors in this matter, regarding both the process and the law itself. This work concludes that there is evidence of an inconsistency between the spirit of the law and the actual attributions of the cosocs.
\end{abstract}

Keywords: New public service, public participation, Chile, social control, Community Councils of Civil Society.

-Profesora asociada del Departamento de Administración Pública y Ciencia Política de la Universidad de Concepción, Chile. ORCID:0000-0002-1087-505X_wmunoz@udec.cl Fecha de recepción: 03 de octubre de 2017. Fecha de aceptación: 12 de marzo de 2018. 


\section{Introducción'}

Después de las reformas posburocráticas implementadas en la década de los setenta en las administraciones públicas, que tenían como finalidad combatir el déficit fiscal y dar respuesta a las demandas de los propios ciudadanos, diversos autores intentaron sistematizarlas dándoles distintos nombres, como paradigma posburocrático-idea de Michael Barzelay-, Gobierno empresarial, emprendedor - por Oborne y Gaebler-, gerencialismo -por Pollit-, Gobierno por mercado -por Self-, y el más conocido, nueva gestión pública -por Hood- (Aguilar, 2006, p. 144). Una de las principales características de estas reformas fue que elevaron la categoría del hasta entonces usuario, convirtiéndolo en cliente y dando importancia a su opinión en cuanto a la satisfacción por la recepción de prestaciones públicas.

Entre la década de los ochenta y noventa, este nuevo paradigma se intentó implementar en América Latina, aunque sin considerar la poca solidez institucional que tenía la región. La falta de democracia, presente por muchas décadas en la mayoría de sus países, afectó a sus administraciones públicas, dejándolas a merced de quienes ostentaban el poder y afectando a aquellas instituciones en las que el Estado concretiza sus fines.

Muchas de estas reformas, algunas realizadas por imposición para recibir préstamos de organismos internacionales (Ramió y Salvador, 2005), tuvieron consecuencias no deseadas para los receptores de la acción pública, por lo que la implementación del concepto de cliente por parte de las administraciones públicas tendría un retraso. El déficit

I. Este trabajo ha sido desarrollado en el marco del Proyecto VRID-Iniciación Código 2 I 8.058.008-I.OIN de la Universidad de Concepción, Chile. Se agradece la colaboración para este artículo de Herman Durán y los estudiantes de la sección 01, año 2017, de la asignatura de Administración Pública de la carrera de Administración Pública, así como del Fondo de Incentivo a la Publicación de la Facultad de Ciencias Jurídicas y Sociales de la Universidad de Concepción. 
democrático presente en muchos países de la región obstaculizaba también la consolidación de una institucionalidad sólida que permitiera darle continuidad a las reformas. En este sentido, los países de América Latina fueron poco a poco transitando hacia Gobiernos democráticos, y con ello adaptando sus administraciones públicas a los nuevos tiempos, sin embargo, no se consideraron las particularidades propias de la región.

Por lo anterior, en 1998 surgió una adaptación a las propuestas posburocráticas con el fin de considerar dicha realidad y comenzar a recibir efectos positivos de las reformas implementadas, aún no percibidos por los ciudadanos de la época. Estas propuestas quedaron plasmadas en el documento doctrinario Nueva gestión pública para América Latina del Centro Latinoamericano de Administración para el Desarrollo (CLAD) (Centro Latinoamericano de Administración para el Desarrollo, 1998). ${ }^{2}$ Desde ese momento

[...] comienza, en América Latina, una nueva ronda de transformaciones en el aparato estatal y en la administración pública. Estos cambios constituirían una agenda más orientada al mejoramiento de la gestión, con gran énfasis en la efectividad de las políticas públicas, en el fortalecimiento de la función pública, en la democratización de la administración pública y en el montaje de una red de políticas sociales, con el objetivo de alcanzar una verdadera ciudadanía social (Centro Latinoamericano de Administración para el Desarrollo, 2010, p. 7).

Lo iniciado en ese momento tuvo como finalidad atender a los ciudadanos desde una perspectiva de cliente, según sus necesidades y requerimientos, considerando que estos estaban siendo cada vez más exigentes y demandantes. 


\section{Hacia el nuevo servicio público}

Ramió y Salvador (2005) señalan que las reformas asociadas a la nueva gestión pública obedecían a dos corrientes:

1) Una neoempresarial, que tenía una hegemonía en sus reformas, visiones y discusiones teóricas, en la que la relación entre las administraciones públicas y sus ciudadanos se convertía en la existente entre una empresa y su cliente.

2) Una neopública, que destacaba el rol del ciudadano, más allá de una simple reconsideración del mismo de usuario a cliente que hacían las corrientes neoempresariales.

La corriente neopública, débil desde finales de los noventa e inicios del siglo XXI, se caracterizaba por lo siguiente:

- Ampliaba la concepción de ciudadanía, con el fin de que esta tradujera su opinión de manera activa en el ámbito de lo público: "Se considera que el concepto de ciudadanía debe servir para, mediante un discurso abierto y pluralista, recrear la legitimidad política en la administración pública" (Ramió y Salvador, 2005, p. 24), garantizándoles nuevos derechos a los ciudadanos.

- Permeaba en los funcionarios los valores públicos, logrando plasmar una cultura en que se privilegiara la eficacia, eficiencia y ética al interior de la gestión pública.

- Priorizaba la satisfacción del ciudadano por la recepción de prestaciones de servicios de diversa índole.

- "[Buscaba] Actuar con principios y valores, como la universalidad y la igualdad, en los servicios públicos[,] contrapuestos a los de economicismo y gestión" (Ramió y Salvador, 2005, p. 25).

- Mejoraba la calidad y cantidad de los servicios públicos.

Con el paso del tiempo, la corriente neopública se transformó en el nuevo servicio público, un nuevo modelo teórico

\section{6}


que buscaba transformar nuevamente la gestión de las administraciones públicas. El nuevo servicio público concibe el rol del ciudadano con un mayor protagonismo. En esta línea, la participación ciudadana en la gestión pública se reconoce como necesaria: los ciudadanos participan en las decisiones públicas, dejando atrás el rol pasivo del usuario y el instrumental del cliente.

Esta nueva etapa en materia de gestión pública, que incluye, pues, una reconfiguración del ciudadano, tiene como consecuencia que se hacen más democráticas las administraciones públicas.

En este sentido, y con la finalidad de garantizar dicha democratización, Denhardt y Denhardt (2007) plantean como desafío a las administraciones cumplir con una serie de principios, resumibles en: 1) servir a los ciudadanos, no a clientes; 2) buscar el interés público; 3) valorar a la ciudadanía sobre el espíritu empresarial; 4) pensar estratégicamente, actuar democráticamente; 5) servir antes que dirigir; y 6) valorar a la gente, no sólo la productividad.

Un último principio, que es importante explicar, es reconocer que la rendición de cuentas no es simple, por lo que, si bien la responsabilidad primera es de las administraciones públicas, es importante que los ciudadanos asuman que para ser partícipes en las decisiones públicas deben estar debidamente informados y ser competentes en las materias en las cuales participarán. Dado que toda decisión pública tiene efectos en una comunidad entera, se debe trabajar bajo una lógica de responsabilidades compartidas, aun cuando la administración pública sea la principal responsable.

Pasar al nuevo servicio público requiere de un cambio de paradigma, en que hay que dar cabida a la ciudadanía en las decisiones públicas. Se debe disponer de manera previa de una sociedad con la suficiente madurez, formada en cuanto a sus derechos y sus obligaciones, y que además cuente con las competencias para poder participar en las decisiones en 
las que quiere aportar y para asumir como obligación las responsabilidades que significa tomar dichas decisiones públicas, en cuya construcción ha sido parte importante.

\section{Participación ciudadana en la gestión pública}

La participación en los asuntos públicos ya se declaraba desde que los intereses generales cobraron importancia a finales del siglo XVIII, cuando los hombres se plantearon en la Declaración de los derechos del hombre y del ciudadano (1789) como constructores de su propia felicidad. Además, la declaración señalaba en su artículo 6: "La ley es expresión de la voluntad de la comunidad. Todos los ciudadanos tienen derecho a colaborar en su formación, sea personalmente, sea por medio de sus representantes [...]" (Asamblea Nacional Francesa, 1789).

Un siglo después, Wilson, asimilando esta participación a la opinión pública, se preguntaba: “¿Qué parte debe tomar la opinión pública en la conducción de la administración?” (1887, p. 214), es decir, consideraba la importancia de tomar en cuenta la opinión pública, aun cuando la cuestión por definir era el momento en que el ciudadano debía dar su opinión. Además, en ese momento, la preocupación para Wilson era que dicha opinión pública debía estar previamente instruida para aportar en las decisiones públicas, algo que todavía al día de hoy se considera un punto esencial.

En 1948, en la Declaración universal de derechos humanos, se reconocía la importancia de dicha participación al señalar en su artículo 21: "Toda persona tiene derecho a participar en el gobierno de su país, directamente o por medio de representantes libremente escogidos" (Declaración Universal de Derechos Humanos. Resolución 217, 1948).

Dado lo anterior, y al reconocer el cambio de paradigma por parte de las administraciones públicas en la concepción del ciudadano, en 2009 se aprobó la Carta iberoamericana

\section{8}


de participación ciudadana en la gestión pública. ${ }^{3}$ La importancia de este documento es que reconoce el derecho de participación ciudadana en la gestión pública, el cual engloba el derecho de información, participación, asociación y expresión sobre lo público por parte de los ciudadanos, dejando en claro que dicho derecho no corresponde sólo a aquellos a quienes se les reconocen sus derechos de ciudadanía y que hacen uso de ellos, sino a todos aquellos habitantes de un territorio cuya gestión pública les concierna. Es decir, hablar de ciudadano con el derecho a participar de la gestión pública

[...] no está referido a las personas con derechos exclusivos de ciudadanía o de nacionalidad[,] sino a todo habitante con respecto a la gestión pública del país en donde reside [...] (Centro Latinoamericano de Administración para el Desarrollo, 2009, p. 2).

La carta recalca uno de los principios del nuevo servicio público, que tiene que ver con la responsabilidad del ciudadano cuando participa en la gestión pública, ya que este tiene "que contribuir al bien común o interés general de la sociedad" (Centro Latinoamericano de Administración para el Desarrollo, 2009, p. 2), es decir, al igual que con el actuar de los servidores públicos, los ciudadanos deberán subordinar sus intereses particulares y colectivos al interés general cuando hagan uso del "derecho de participación ciudadana en la gestión pública" (Centro Latinoamericano de Administración para el Desarrollo, 2009, p. 2).

Otro punto importante de esta carta es que define la participación ciudadana en la gestión pública como

3. La carta fue aprobada en el marco de la IX Conferencia Iberoamericana de Ministros de Administración Pública y Reforma, celebrada en Lisboa, Portugal, entre el 25 y el 26 de junio de 2009, y luego adoptada por la XIX Cumbre lberoamericana de Jefes de Estado y de Gobierno, celebrada en Portugal entre el 30 de noviembre y el I de diciembre de 2009. 
el proceso de construcción social de las políticas públicas que, conforme al interés general de la sociedad democrática, canaliza, da respuesta o amplía los derechos económicos, sociales, culturales, políticos y civiles de las personas, $y$ los derechos de las organizaciones o grupos en que se integran, así como los de las comunidades y pueblos indígenas (Centro Latinoamericano de Administración para el Desarrollo, 2009, pp. 3-4).

\section{La participación ciudadana en Chile}

En el 2004, fue sometido al Congreso Nacional de Chile un proyecto de ley cuyo espíritu buscaba plasmar el derecho de los ciudadanos a participar en la gestión pública. Luego de años de trámite legislativo, en el año 2011 se aprobó la Ley 20.500 sobre Asociaciones y Participación Ciudadana en la Gestión Pública, que reconoce la importancia de considerar a los ciudadanos en las decisiones públicas, proporcionando distintos mecanismos de participación ciudadana y permitiendo con ello que la ciudadanía se manifieste respecto a temas que son de su interés. Dicha norma introdujo una serie de modificaciones legales, entre ellas a la Ley 18.575 de Bases de la Administración General del Estado y la Ley 18.695 Orgánica Constitucional de Municipalidades.

En el caso de la Ley 18.575, se agregó, por instrucción del artículo 32 de la ley 20.500, el título IV, "De la participación ciudadana en la gestión pública", con artículos que reconocen el derecho de los ciudadanos a participar en "sus políticas, planes, programas y acciones" (Junta de Gobierno de la República de Chile, 1986), lo que deja a las distintas instituciones públicas la determinación de las formas de participación de los ciudadanos en materia de su competencia (artículo 70). El título obliga, además, a las instituciones a la utilización de medios electrónicos u otros para dar a conocer información de interés público (artículo 71).

Otro punto importante contemplado en la Ley 18.575 es que las instituciones públicas deben dar cuenta pública 
de una manera participativa, con la posibilidad de que la ciudadanía pueda hacer observaciones, planteamientos o consultas, debiendo responderse a estos según la modalidad que se haya establecido previamente (artículo 72). Además, se debe determinar cuáles serán aquellas materias de competencia de la institución pública en las que se solicitará la opinión ciudadana, garantizando que dicha consulta sea informada, pluralista y representativa (artículo 73).

En el caso del artículo 74 de la misma ley, su importancia para el presente trabajo es que señala que

Los órganos de la administración del Estado deberán establecer Consejos de la Sociedad Civil [CSC], de carácter consultivo, que estarán conformados de manera diversa, representativa y pluralista por integrantes de asociaciones sin fines de lucro que tengan relación con la competencia del órgano respectivo (Junta de Gobierno de la República de Chile, 1986).

Es decir, cada institución deberá constituir un consejo que sea representativo de la ciudadanía y que aborde temáticas vinculadas a las funciones del órgano público. En este sentido, una forma en que se institucionaliza la participación ciudadana es a través del CSC, el que una vez constituido nombra su presidente de entre sus miembros.

Resumiendo, de los artículos modificados en esta Ley 18.575, se desprende que, además de los CSC, las instituciones públicas deben implementar otros mecanismos para que los ciudadanos pueden participar, como lo son las cuentas públicas participativas, la transparencia pasiva y la consulta ciudadana:

- Las cuentas públicas participativas se relacionan con que las instituciones públicas deben transparentar su accionar, lo que permite el control social por parte de los ciudadanos. Por lo tanto, dichas cuentas constituyen "un mecanismo propio de un Estado democrático 
y alude[n] al anglicismo 'accountability" (División de Organizaciones Sociales, 2014, p. 31). Así, los servicios públicos están obligados anualmente a rendir cuentas, tras lo cual la ciudadanía puede formular consultas y observaciones que deben ser respondidas por la autoridad respectiva, debiendo transformarse esta dinámica en un diálogo entre la entidad pública y los ciudadanos. En este sentido, la División de Organizaciones Sociales (DOS) (2014) es tajante al señalar que las cuentas públicas participativas no son actividades en que sólo se entrega o publica información a través de las páginas web institucionales, ya que la esencia de estas cuentas es el diálogo entre las instituciones públicas y la sociedad civil. ${ }^{4}$

- La transparencia pasiva es un mecanismo por el cual el ciudadano puede ejercer el derecho a solicitar información pública, reconocido en el 2008 con la publicación de la Ley 20.285 sobre Acceso a la Información Pública (Congreso Nacional, 2008). Lo importante de este derecho es que con la información obtenida los ciudadanos pueden ejercer de mejor manera otro derecho: participar de la gestión pública de manera informada. Para lo anterior, las instituciones deben disponer en sus páginas web de un ícono de transparencia pasiva, para que el ciudadano pueda solicitar la información a través de él. También, el ciudadano puede solicitar la información de manera presencial en la institución pública.

- Las consultas ciudadanas hacen referencia al proceso "a través del cual se somete[n] a consideración de la ciudadanía temas de interés público, mediante distintas herramientas que permiten informar y recoger los distintos puntos de vista, perspectivas y opiniones, a objeto 
de ser considerados en los distintos momentos del ciclo de las políticas públicas" (División de Organizaciones Sociales, 2014, p. 20). Las consultas se pueden realizar de manera presencial, como diálogos participativos, o de manera virtual, como consultas ciudadanas virtuales.

Si bien la Ley 20.500 se aprobó en 2011, hasta agosto de 2014 los CSC aún no se constituían en su mayoría, por lo que, sobre este y otros puntos pendientes en materia de participación ciudadana, la presidenta de la república, Michelle Bachelet, ${ }^{5}$ dictó el Instructivo presidencial no. 7, en el cual, al iniciar el punto 1, reconocía los cambios vividos en el país, señalando que en la actualidad hay

[...] una mayor conciencia ciudadana en el ejercicio de los derechos y en una creciente demanda de participación. La conducción de los asuntos públicos hoy no se concibe sin el involucramiento activo de los ciudadanos en las diferentes facetas de la política pública y, por lo tanto, resulta imprescindible desarrollar nuevas estrategias de vinculación entre el Estado y sociedad civil que de[n] cuenta de este escenario [...] (Presidencia de la República, 2014).

Lo anterior sería el preámbulo que haría la presidenta para exigir a las instituciones públicas en el mismo instructivo la implementación, en un plazo de seis meses, de las normas relativas a: la participación ciudadana; la creación de la Unidad de Participación Ciudadana, los CSC y la cuenta pública participativa; así como la puesta en marcha del Registro Nacional de Personas Jurídicas sin Fines de Lucro.

El instructivo, en su numeral 6, aumentó los mecanismos para hacer efectiva la participación de los ciudadanos en la gestión pública, agregando a la Audiencia Pública, los presupuestos participativos, los Cabildos Ciudadanos Territoriales y Sectoriales, los Encuentros de Diálogos

5. En su segundo mandato presidencial, correspondiente al periodo 20I4-20I8. 
Participativos y las Plataformas Digitales Participativas. Al día de hoy, el desafío es que dichos mecanismos sean puestos en práctica.

En el ámbito de lo local, la Ley 20.500 también introdujo modificaciones en la Ley 18.695 Orgánica Constitucional de Municipalidades, al incorporar la participación de la sociedad civil a través del Consejo Comunal de Organizaciones de la Sociedad Civil (COSOC), el cual se compone, según el artículo 94, por

[...] organizaciones comunitarias de carácter territorial y funcional, y por las organizaciones de interés público de la comuna. Asimismo, y en un porcentaje no superior a la tercera parte del total de sus miembros, podrán integrarse aquellos representantes de asociaciones gremiales y organizaciones sindicales, $o$ de otras actividades relevantes para el desarrollo económico, social y cultural de la comuna (Junta de Gobierno de la República de Chile, 1988).

Los miembros electos del COSOC durarán en sus cargos cuatro años. A diferencia de los CSC, los COSOC son presididos por el alcalde o alcaldesa de un municipio, ya que el artículo 63, letra m, de la Ley 18.695 le da como atribución a esta autoridad "convocar y presidir" el consejo (Junta de Gobierno de la República de Chile, 1988). Esta condición pone en una situación incómoda a los miembros de los COSOC que son representantes de las distintas organizaciones, ya que al ser presididos por el alcalde o alcaldesa se encuentran en una situación de subordinación, lo que claramente atenta con el espíritu de la ley, que reconoce el principio participativo "como una cooperación entre ambos y no como una relación vertical o de sumisión de los sujetos a la autoridad" (Junta de Gobierno de la República de Chile, 1988). 


\section{Hacia el empoderamiento de los cosoc}

Como ya se señaló, a partir del 2011 se debían constituir los COSOC. Sin embargo, el proceso ha sido lento: al año 2017 no se encontraban constituidos en los trescientos cuarenta y cinco municipios del país.

En la región del Biobío, en el 2013 y a iniciativa de una corporación privada, el Servicio Evangélico para el Desarrollo (SEPADE), se constituyó una mesa provincial de seguimiento de la implementación de la Ley 20.500. ${ }^{6}$ En 2014, a iniciativa de la mesa provincial, se realizó el Primer Congreso Regional de Consejeros de la Sociedad Civil del Biobío, con una asistencia de ciento treinta y ocho consejeros de la sociedad civil representando a veintitrés de las cincuenta y cuatro comunas de la región ${ }^{7}$ (Mesa de seguimiento Ley 20.500 región Biobío, 2014). Sin embargo, ningún presidente de estos consejos, es decir, ningún alcalde o alcaldesa, asistió. ${ }^{8}$

Como resultado de este congreso, surgieron las siguientes propuestas cuya solución, en su mayoría, tendría que hacerse a través de una modificación a la Ley 20.500 (Mesa de seguimiento Ley 20.500 región Biobío, 2014):

- Que el presidente de los COSOC sea electo democráticamente de entre los miembros que representan a

6. Conformada por representantes de la Universidad de Concepción, la corporación SEPADE, SEDEC, la Municipalidad de Talcahuano, un representante de la División de Organizaciones Sociales y consejeros de las siete comunas de la provincia de Concepción (Mesa de seguimiento Ley 20.500 región Biobío, 20I4). 7. El 5 de septiembre de 2017 se aprobó la Ley 21.033 , que creó la región de Nuble. Dicha ley entraría en vigencia en septiembre de 2018. Con ella, se redujo el número de comunas de la región del Biobío de cincuenta y cuatro a treinta y tres. Las veintiuna restantes serán incorporadas a la nueva región de Nuble. 8. La única excepción fue la del alcalde de la llustre Municipalidad de Santa Juana, Ángel Castro. Sin embargo, este no asistió como miembro del cosoc, sino como presidente de la Asociación Regional del Biobío. Además, la municipalidad de Santa Juana, a la fecha de elaboración del congreso, aún no tenía constituido su COSOC, lo que haría hasta el 12 de septiembre de 2016 . 
la sociedad civil, dejando sin efecto la titularidad del alcalde o alcaldesa.

- Que al momento de redactar un proyecto que modifique la Ley 20.500, sea considerada la opinión de los representantes de los COSOC.

- Que la ley debe considerar un organismo autónomo, nacional y representativo que fiscalice el cumplimiento y la implementación de los COSOC.

- Que se consideren recursos presupuestarios en la municipalidad exclusivos para el funcionamiento e implementación de los COSOC.

- Que las decisiones que adopte el consejo sean de carácter vinculante y de alcance comunal -lo que no está contemplado en la actual legislación-.

- Que todas las consideraciones anteriormente señaladas podrían ser las bases para la eventual creación de una institucionalidad administrativa de carácter público, posiblemente un Ministerio de Participación Ciudadana o entidad similar.

La reflexión dada durante esa jornada fue considerada en análisis posteriores al interior de la propia mesa de seguimiento de la Ley 20.500. Otro trabajo realizado en este sentido se dio en septiembre de 2015, cuando la DOs de la región del Biobío realizó un diálogo participativo con la presencia de ciento once miembros de distintos Cosoc. De este encuentro, se obtuvo la visión de lo que los miembros esperan respecto a la participación ciudadana, lo que se resume en:

- Que la participación ciudadana sea reconocida en la propia Constitución, fortaleciendo así su institucionalización.

- Que se le reconozca a la participación ciudadana un carácter vinculante respecto a sus propuestas. 
- Que cuente con presupuesto y se aumente el Fondo de Fortalecimiento de Fondos de Interés Público, creado por la Ley 20.500.

- Que se establezca el Consejo Nacional de Participación Ciudadana de Chile.

- Que se contemple un seguro de vida o accidente, un tema no menor, para los dirigentes, u otros incentivos, ya que hay un tiempo destinado al trabajo en participación ciudadana que tiene sus costos, si bien dicho trabajo se realiza por compromiso y convicción (División de Organizaciones Sociales, Ministerio Secretaría General de Gobierno, y Gobierno de Chile, 2015).

El segundo congreso regional de COSOC del Biobío, celebrado el 21 de noviembre de 2015, reconoció la importancia de visibilizar el trabajo de los representantes de los COSOC y empoderarlos más respecto a sus derechos, por lo que dicha instancia tuvo como principal objetivo conformar la Asociación Regional de COSOC del Biobío (ACosoc Biobío).

Al año siguiente, en abril de 2016, el empoderamiento ciudadano se profundizaría aún más con la conformación de la Federación Nacional de Consejeros de la Sociedad Civil (CHILECOSOC), ${ }^{9}$ con un directorio provisional que luego, en agosto de 2016, sería elegido definitivamente, asumiendo la presidencia Juan Carlos Venegas.

A comienzos del mismo año, y como el Instructivo presidencial no. 7, ya mencionado, establecía la creación de un Consejo Nacional de Participación Ciudadana y Fortalecimiento de la Sociedad Civil, ${ }^{10}$ se dictó el Decreto 001, con

9. Realizada en la Sala del Senado del Ex Congreso Nacional, en Santiago. I0. El artículo 4 del decreto señala que será consultivo y sus integrantes permanecerán en funciones por el periodo de un año a partir de la fecha de constitución, por lo que al final de su mandato, en enero de 2017, el consejo debía entregar el primer informe sobre el estado de la participación ciudadana en Chile, y propuesta de estándares para evaluar en el futuro su nivel de avance (Consejo Nacional de Participación Ciudadana y Fortalecimiento de la Sociedad Civil, 2017). 
fecha del 14 de enero de 2016, que daba vida a dicho consejo, cuya finalidad sería:

[...] asesorar a la presidenta de la república en materia de institucionalización de la participación ciudadana y el fortalecimiento de la sociedad civil en el país, en el marco de un amplio debate sobre la materia (Presidencia de la República, 2016).

El consejo quedó conformado por distintos actores de la sociedad civil, trabajó durante todo el 2016 y concluyó con un informe que se le hizo llegar a la presidenta de la república con fecha 17 de enero de 2017.

El consejo realizó un trabajo arduo a lo largo del país, recogiendo la mirada que los actores de la sociedad civil tenían respecto a la participación ciudadana, las trabas que presenta la ley y las limitaciones que los mismos actores reconocen para ser verdaderos aportadores a la gestión pública.

Los resultados de este trabajo no fueron muy diferentes a los presentados en el primer congreso COSOC de la región del Biobío y el dialogo participativo organizado por la DOS en la región del Biobío, ya mencionados. Al respecto, podemos citar:

La Ley 20.500 carece de mecanismos de fiscalización y sanción que aseguren su cumplimiento, dejándolo a criterio y voluntad del directivo del servicio.Tampoco se establece el financiamiento de los mecanismos de participación de modo de garantizar su funcionamiento estable. El carácter de estos mecanismos como consultivos, informativos y no vinculantes los hace muy poco efectivos, lo que desmotiva a muchos dirigentes a participar en ellos. También la Ley 20.500 exceptuó a los Gobierno regionales de las obligaciones en materia de participación ciudadana (Consejo Nacional de Participación Ciudadana y Fortalecimiento de la Sociedad Civil, 2017, pp. 29-30).

\section{8}


Sistematizada toda la información recogida, este consejo nacional realizó una serie de propuestas, siendo una de ellas muy importante por su vínculo con la definición normativa de ciudadano, ya que planteó dejar atrás la visión actual, que es restrictiva. ${ }^{11}$ Dicha propuesta sería modificar o considerar en una eventual nueva carta magna una definición más amplia de ciudadano que considere a "todos los miembros de la sociedad como personas capaces de participar de las deliberaciones" (Consejo Nacional de Participación Ciudadana y Fortalecimiento de la Sociedad Civil, 2017, p. 42), es decir, se sugiere considerar a aquellas "personas que habitan en Chile como ciudadanos de su Estado para todos los efectos, y que luego la distinción para efectos electorales se haga en una mención aparte y con otra denominación" (Consejo Nacional de Participación Ciudadana y Fortalecimiento de la Sociedad Civil, 2017, p. 42). Lo anterior, de aprobarse, permitiría adecuar la normativa chilena a lo planteado en la ya mencionada Carta iberoamericana de participación ciudadana en la gestión pública.

Retomando la constitución de los COSOC en la región del Biobío, se puede señalar que esta sigue siendo una tarea pendiente, ya que según los datos obtenidos entre el 2016 y el 2017, apenas poco más del 60\% de los municipios de la región tiene vigentes sus Cosoc.

II. El artículo 13 de la constitución política de Chile de 1980 dice que son ciudadanos los chilenos que hayan cumplido dieciocho años de edad y que no hayan sido condenados a pena aflictiva. 
Tabla 1. Constitución de COSOC años 2016-2017

\begin{tabular}{ccrcr} 
Situación & \multicolumn{2}{c}{ Año 2016} & \multicolumn{2}{c}{ Año 2017 } \\
Constituidos & 33 & $61 \%$ & 35 & $65 \%$ \\
No constituidos & 21 & $39 \%$ & 19 & $35 \%$ \\
\hline
\end{tabular}

Fuente: elaboración propia con datos particulares proporcionados por ACOSOC Biobío y los sistemas de transparencia activa y pasiva de los municipios. La información está en poder de la autora del presente artículo.

Los datos muestran que aún hay camino por recorrer para que el mecanismo de participación que son los COSOC sea, por una parte, constituido, y por otra se consolide su conformación, ya que en algunos casos, habiendo sido constituidos, no han sido renovados con el tiempo. Un caso emblemático de esto es Hualqui, comuna pionera en constituir su COSOC y que luego no logró consolidarlo y pasó a transformarse en una de las comunas sin COSOC constituido.

Figura 1. Constitución de COSOC años 2016-2017

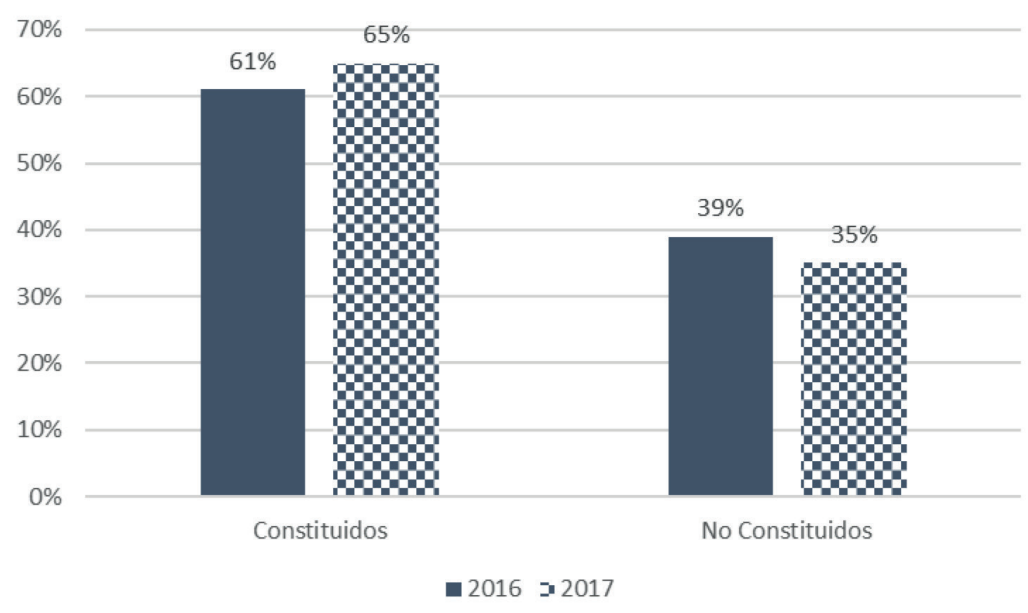

Fuente: elaboración propia con datos particulares proporcionados por ACOSOC Biobío y los sistemas de transparencia activa y pasiva de los municipios. La información al respecto está en poder de la autora del presente artículo. 
La Figura 1 muestra un leve aumento en la constitución de los COSOc, pero esta sigue siendo insuficiente a seis años de la publicación de la Ley 20.500. De las municipalidades que no tienen constituido su COSOC, como Alto Biobío, se precisa que

[...] en su estructura organizacional, de acuerdo a la Ley Indígena 19.253, las formas de participación de la comunidades en general son a través de las organizaciones denominadas Comunidades y Asociaciones Indígenas, razón por la cual existe como sustituto del COSOC un directorio conformado por autoridades tradicionales compuestas por lonkos y presidentes de comunidades indígenas (Municipalidad de Alto Biobío, 2017).

Lo anterior obliga a considerar, en eventuales reformas a la ley, situaciones como la recientemente expuesta.

\section{Visión de los actores}

La publicación de la Ley 20.500 no trajo la solución mágica para generar una sociedad partícipe de los asuntos públicos. En esa línea, se debe ir avanzando para transformar a las administraciones públicas, dando los espacios necesarios para que los ciudadanos puedan participar en su gestión, y a su vez estos mismos deben prepararse para constituir un aporte real.

En este caminar, es importante ir conociendo los desafíos que ofrece la ley y el trabajo mismo que han ido realizando distintos actores. En este sentido, se ofrece a continuación la visión de cuatro actores vinculados a la cuestión: la encargada de la DOs de la región del Biobío, el presidente de CHILECosoc, la encargada de Participación Ciudadana 
de la Ilustre Municipalidad de San Pedro de la Paz $^{12}$ y el presidente de la Asociación de Municipalidades de la Región del Biobío. ${ }^{13}$

Para estos actores, la Ley 20.500 tiene como gran fortaleza haber marcado un hito en materia de participación ciudadana de la gestión pública. Para el presidente de CHILECOSOC, esta ley es una gran oportunidad, ya que "permite instalar de alguna forma, el derecho a la participación ciudadana como un derecho" (entrevista personal, 2017c); aun cuando se critique el contenido de la ley, su importancia radica en que deja instalado el tema de la participación ciudadana.

Esta idea también es compartida por muchos consejeros de Cosoc de la región del Biobío, los que en el 2014 concluyeron en su primer congreso que la participación ciudadana ya es una realidad y que "ha llegado para quedarse, esto porque ya está clara la decisión de avanzar en profundizar y mejorar este mecanismo, dado que se presenta como una oportunidad de incidir en el desarrollo local de las comunas" (Mesa de seguimiento Ley 20.550 región Biobío, 2014).

A pesar de lo positivo de la Ley 20.500, se le reconocen muchos problemas. Uno de ellos es el hecho que los COSOC sean presididos por el alcalde o alcaldesa, agregando además, la discrecionalidad que tiene esta autoridad para convocar a las sesiones, por la cual puede llegar a suceder que por mucho tiempo no sean convocadas.

En esta línea, debe recordarse que ya en el primer congreso de COSOC se discutía este tema, siendo la primera de las propuestas surgidas que el presidente de los COSOC fuera electo entre los representantes de la sociedad civil. De

12. Las entrevistas semiestructuradas a la encargada de DOS, el presidente de CHILECOSOC y la encargada de participación de la Municipalidad de San Pedro fueron realizadas entre los meses de marzo y abril de 2017.

13. La entrevista fue estructurada y realizada por medio de correo electrónico en marzo de 2018. 
no modificarse la actual presidencia de los COSOC, habría que preguntarse ¿cómo se puede ejercer un control social si quien preside un órgano de la sociedad civil es la misma autoridad pública? Sin duda, la respuesta es que sería muy difícil ejercer dicho control.

Otro punto a destacar es que para la encargada de la DOS entrevistada, el trabajo que se da en la actualidad con los distintos consejos -tanto CSC como COSOC- depende de la "persona que esté a cargo de la participación" (entrevista personal, 2017a), lo que debería depender, más bien, por una parte, de la normativa que esté alineada y promueva dicha participación, y por otra parte, del compromiso de los representantes de la sociedad civil llamados a formar parte de estos consejos.

Tal cual está la ley hoy día, en ella cobra importancia la voluntad política de la propia autoridad para implementar cualquier mecanismo, ya que la normativa permite y ha permitido que muchos de los mecanismos no se hayan implementado o, en otros casos, de haberse implementado, que lo hayan hecho de forma muy cercana a lo mínimo que la ley exige. Esto se reafirma por lo planteado por la encargada de Participación Ciudadana de San Pedro de la Paz, quien señala que la implementación de uno de los mecanismos "tiene mucho que ver con la voluntad política de los Gobiernos locales de constituir el tema de los COSOC" (entrevista personal, 2017b).

En este sentido, al tener muchos vacíos la Ley 20.500, y dado el hecho de que "tanto en las élites políticas chilenas como en la administración pública" hay "una actitud poco proclive a la participación vinculante" (Consejo Nacional de Participación Ciudadana y Fortalecimiento de la Sociedad Civil, 2017, p. 36), las experiencias positivas en materia de participación ciudadana en la gestión pública obedecen a la voluntad de la propia autoridad pública y no a un real reque- 
rimiento normativo que permita garantizar el ejercicio del derecho a la participación ciudadana en la gestión pública.

Los ejemplos de buenas prácticas en materia de participación ciudadana, como lo es el caso de la municipalidad de San Pedro de la Paz, que lleva ya siete años trabajando con los ciudadanos de esa comuna cuestiones como los presupuestos participativos, han sido posibles gracias al convencimiento de los propios alcaldes sobre la importancia de considerar la opinión de los ciudadanos en las decisiones presupuestarias. En el caso de San Pedro de la Paz, en el 2017 incluso dio un paso más allá, al considerar como ciudadanos a los menores de edad al implementar los presupuestos participativos infantiles. ${ }^{14}$

Aun así, se hace necesaria una "modificación a la ley" que "tiene que ver con los mecanismos, tiene que ver con los medios de fiscalización, para que esta ley sea cumplida, con el financiamiento, tiene que ver con aspectos normativos [...]" (entrevista personal, 2017c). Vale señalar que la Ley 20.285 sobre Acceso a la Información Pública, que reconoce y garantiza el derecho al acceso a esta, contempló en su artículo 31 la creación de una corporación autónoma, denominada Consejo para la Transparencia que sí tendría dichas facultades. ${ }^{15}$ Sin embargo, la Ley 20.500 no contempló la creación de un órgano de esta naturaleza en materia de participación ciudadana. Recordemos que este punto ha sido un problema reconocido en distintas instancias, lo que

14. En el 2017, el municipio incorporó a la participación ciudadana a ciudadanos de entre seis y catorce años que propusieron diez ideas para que fueran financiadas por el municipio y que luego serían parte de los proyectos que ellos mismos priorizarían a través de una votación, realizada el día I I de septiembre del mismo año con la participación de unos cuatrocientos ciudadanos de la misma edad (Municipalidad de San Pedro de la Paz, 20I I).

I5. Las funciones de este consejo están establecidas en el artículo 32 de dicha ley,y buscan "promover la transparencia de la función pública, fiscalizar el cumplimiento de las normas sobre transparencia y publicidad de la información de los órganos de la administración del Estado, y garantizar el derecho de acceso a la información" (Congreso Nacional, 2008). 
además fue recogido en el informe del Consejo Nacional de Participación Ciudadana y Fortalecimiento de la Sociedad Civil del 2017, al proponer la incorporación de un nuevo título en la ley que contemplara lo siguiente:

5. Créase el Consejo Nacional de Participación Ciudadana y Fortalecimiento de la Sociedad Civil, como una corporación autónoma de derecho público, con personalidad jurídica, patrimonio propio y presencia regional. El consejo tendrá representación regional mediante el establecimiento de Consejos Regionales de Participación Ciudadana.

6. El objeto del consejo será la promoción y el fortalecimiento de la asociatividad y la participación ciudadana en la gestión pública, fiscalizar el cumplimiento de las normas de participación ciudadana y garantizar el derecho a la asociatividad y a la participación ciudadana en la gestión pública [...] (Consejo Nacional de Participación Ciudadana y Fortalecimiento de la Sociedad Civil, 2017, p. 44).

Otro punto importante es el compromiso del ciudadano con su comunidad, y esto hay que asumirlo como un desafío necesario para que se obtengan buenos frutos de la participación ciudadana. En este sentido, es clave el ciudadano, en tanto es el que conoce mejor la realidad local para la cual gestiona una municipalidad. Al respecto, el presidente de la Asociación de Municipalidades de la Región del Biobío cree que existe ese compromiso y señala:

Los ciudadanos están preparados hoy más que nunca para realizar aportes a la gestión municipal, considerando que son quienes mejor conocen su contexto. Lo importante es que existan canales de comunicación para aquello, de ahí el rol fundamental del cosoc. Lo que falta es una mayor difusión, para que los ciudadanos conozcan los cosoc (entrevista personal, 2018).

Para la encargada de Participación Ciudadana de la Municipalidad de San Pedro de la Paz, también es evidente la falta 
de difusión de la que habla el presidente de la Asociación de Municipalidades de la Región del Biobío, ya que a pesar de que "se hizo esta ley [en] el 2011, recién este año 2017 [los ciudadanos] tiene[n] o ha[n] escuchado [sobre] este tema o tiene[n] alguna noción", lo que le permite concluir que "no creo que estén preparados" (entrevista personal, 2017b) para tomar decisiones en conjunto con las administraciones locales, planteamiento distinto a lo señalado por el presidente de la Asociación de Municipalidades de la Región del Biobío, quien sí cree que los ciudadanos están preparados para aportar a las decisiones. En este sentido, se puede señalar que aun cuando los ciudadanos puedan desconocer la ley, conocen la realidad que ellos viven, y dicha realidad es importante hacerla saber a las autoridades pues el éxito de las decisiones que se tomen puede relacionarse con la consideración de esa realidad desde la mirada de sus propios protagonistas.

Otras de las debilidades percibidas es que la Ley 20.500 es "muy amplia y vaga en comparación a la [Ley] 19.418, ${ }^{16}$ que tiene claramente definido[s] cuales son los roles y funciones de la directiva y de sus integrantes como socios, no así la 20.500, que sólo da instrucciones de cómo se constituye, y muy a grandes rasgos, lo que debería ser el COSOC, pero no da funciones para el COSOC" (entrevista personal, 2017b). En este sentido, cualquier reforma de ley debería contemplar una definición más clara de los roles de los distintos actores reconocidos en la Ley 20.500, entre ellos los miembros del cosoc.

Respecto a las propuestas para mejorar esta ley, como la que está actualmente en manos de la Presidencia de la República y que busca perfeccionarla, al parecer estas no han contemplado a todos los involucrados, ya que el presidente de la Asociación de Municipalidades de la Región del Biobío 
es categórico al señalar que "no hemos sido considerandos como actores en estos planteamientos" (entrevista personal, 2018), sin embargo, sus propuestas son coincidentes con las entregadas a la Presidencia de la nación, pues señala que

[...] se debe dotar de mayores recursos para la implementación de esta ley y de capacidad de fiscalización para su cumplimiento, además se debe sancionar, por ejemplo, el no tener ordenanza de participación. Además, se debe[n] validar los consejos que existen y articularlos a nivel territorial (entrevista personal, 2018).

Aunque estas propuestas estarían siendo abordadas en una eventual modificación legal, es necesario señalar que en el trabajo de levantamiento de información sobre problemas y sus soluciones siempre se debe abarcar a todos los actores, ya que la participación ciudadana en la gestión pública no sólo debe contemplar a los ciudadanos, sino a todos los que de una u otra forma están involucrados en la gestión de los asuntos públicos.

\section{Conclusiones}

El nuevo servicio público considera al ciudadano participante en las decisiones públicas. En Chile, se ha ido avanzando en esta materia con la Ley 20.500, la que entre otras cosas da vida a la participación ciudadana a través de los COSOc. La enseñanza que nos ofrecen los consejos comunales, que se han constituido en asociaciones regionales (ACOSOC Biobío) y hace un año en una federación nacional (CHILECOSOC) para alzar la voz y exigir mayores atribuciones, da elementos tangibles para afirmar que ya se inició el empoderamiento de estos, y que con ello se podrá ofrecer al resto de la ciudadanía el camino hacia la participación en temas públicos. Si bien por el momento los consejos sólo cumplen un rol consultivo, se ve en los actua- 
les representantes de los COSOC un compromiso con esta nueva forma de participación en cuanto al asociacionismo y el empoderamiento ciudadano. Por parte de las instancias públicas, hay un compromiso con fortalecer las capacidades de la ciudadanía, es decir, de prepararla para incorporarse en la discusión.

Como ya se ha señalado, se tiene pendiente el hecho de que no se haga obligatoria la adopción de decisiones respecto a los pronunciamientos ciudadanos, lo que deja débil el tránsito hacia este nuevo enfoque en materia de gestión. Sin embargo, ya se les han entregado atribuciones a los ciudadanos que si bien son pocas, son semillas que pueden germinar en el empoderamiento ciudadano para participar en las decisiones de carácter público que tienen o tendrán impacto en ellos. En esta línea, se espera que el informe presentado por el Consejo Nacional de Participación Ciudadana y Fortalecimiento de la Sociedad Civil sea considerado en las discusiones que se den en torno a próximas modificaciones legales al respecto de esta problemática.

Finalmente, se puede concluir que la normativa chilena, en su espíritu, reconoce la importancia de la participación ciudadana en la gestión pública. Respecto a si ha sido la institucionalidad la que ha empoderado a la ciudadanía, se considera que no es así: han sido los propios ciudadanos los que se han ido empoderando y alzando su voz en esta materia.

Se puede señalar que el nuevo servicio público en Chile ya inició su camino. El tiempo dirá si fue el correcto. 
Aguilar, L. (2006). Gobernanza y gestión pública. México: Fondo Bibliografía de Cultura Económica.

Asamblea Nacional Francesa (1789). Declaración de los derechos del hombre y del ciudadano. Recuperado de: http:// www.conseil-constitutionnel.fr/conseil-constitutionnel/ root/bank_mm/espagnol/es_ddhc.pdf

Centro Latinoamericano de Administración para el DesarroIlo (1998). Nueva gestión pública para América Latina. S/l: CLAD. Recuperado de: http://old.clad.org/documentos/ declaraciones/una-nueva-gestion-publica-para-americalatina/view

Centro Latinoamericano de Administración para el DesarroIlo (2009). Carta iberoamericana de participación ciudadana en la gestión pública. Lisboa: Centro Latinoamericano de Administración para el Desarrollo. Recuperado de: http://old.clad.org/documentos/declaraciones/cartaiberoamericana-de-participacion-ciudadana/view

Centro Latinoamericano de Administración para el Desarrollo (2010). Gestión pública iberoamericana para el siglo XXI. Santo Domingo: Centro Latinoamericano de Administración para el Desarrollo. Recuperado de: http://old.clad.org/documentos/declaraciones/gestionpublica-iberoamericana-para-el-siglo-xxi/view

Congreso Nacional (2008). "Ley 20.285 sobre Acceso a la Información Pública”. Diario Oficial de la República de Chile. Recuperado de: https://www.leychile.cl/ Navegar?idNorma $=276363$

Congreso Nacional (20 I I)."Ley 20.500 sobre Asociaciones y Participación Ciudadana en la Gestión Pública”. Diario Oficial de la República de Chile. Recuperado de: https:// www.leychile.cl/Navegar?idNorma= 1023 | 43

Consejo Nacional de Participación Ciudadana y Fortalecimiento de la Sociedad Civil (2017). Estado de la participación ciudadana y propuestas de reforma a la Ley 20.500 sobre Asociación y Participación Ciudadana en la Gestión 
Bibliografía
Pública. Santiago de Chile: Consejo Nacional de Participación. Recuperado de: http://consejoparticipacion.cl/ como-funciona-el-consejo

Declaración universal de derechos humanos. Resolución 217 (1948). Recuperado de: http://www.un.org/es/comun/ docs/?symbol=A/RES/2 I7(III)

Denhardt, J., y Denhardt, R. (2007). The New Plubic Service. Serving not Steering. Nueva York: M.E. Sharpe.

División de Organizaciones Sociales (20l4). Criterios y orientaciones para la implementación de mecanismos de participación ciudadana en la gestión pública. Ministerio Secretaría General de Gobierno. Recuperado de: http:// www.dipres.gob.cl/594/articles-87929_Criterios.pdf

División de Organizaciones Sociales, Ministerio Secretaría General de Gobierno, y Gobierno de Chile (2015). Diálogo participativo Ley 20.500, participación ciudadana. División de organizaciones sociales. Recuperado de: http:// www.participemos.gob.cl/wp-content/uploads/20 I 5/I I/ INFORME-DIALOGO-20500-biob\%C3\%ADo.pdf Junta de Gobierno de la República de Chile (1986)." "Ley I8.575, de Bases Generales de la Administración del Estado". Diario Oficial de la República de Chile. Recuperado de: https://www.leychile.cl/Navegar?idNorma=29967\&r= I Junta de Gobierno de la República de Chile (1988). "Ley 18.695, Orgánica Constitucional de Municipalidades”. Diario Oficial de la República de Chile. Recuperado de: https://www.leychile.cl/Navegar?idNorma=30077

Mesa de seguimiento Ley 20.500 región Biobío (II de octubre de 2014). Acta primer Congreso Regional de Consejeros de la Sociedad Civil del Biobío. Concepción, Chile: Congreso Regional de Consejeros de la Sociedad Civil del Biobío.

Municipalidad de Alto Biobío (2017). Informe. Recuperado de: http://www.transparenciaaltobiobio.cl/files/Aclaracion.pdf 
Municipalidad de San Pedro de la Paz (I I de septiembre de Bibliografía $20 \mathrm{II})$. Primera experiencia de Presupuestos Participativos Infantiles. Más de 400 niños decidieron ejecución de 5 proyectos en San Pedro de la Costa. Recuperado de: http:// sanpedrodelapaz.cl/20 I/09/mas-de-400-ninos-decidieron-ejecucion-de-5-proyectos-en-san-pedro-de-la-costa/ Muñoz,W. (2I de marzo de 20I7a). Entrevista personal a encargada de la División de Organizaciones Sociales, región Biobío. Concepción, Chile.

Muñoz, W. (29 de marzo de 20I7b). Entrevista personal a encargada de Participación Ciudadana de San Pedro de la Paz. Ilustre Municipalidad de San Pedro de la Paz, Chile. Muñoz,W. (04 de abril de 20 17c). Entrevista personal al presidente de CHILECOSOC. Universidad de Concepción, Chile. Muñoz,W. (05 marzo de 20I8). Entrevista a presidente de la Asociación de Municipalidades de la Región del Biobío, mediante correo electrónico. Concepción, Chile.

Presidencia de la República (2004). Mensaje presidencial $n^{\circ}$ 48-35 I, dirigido al presidente de la Cámara de Diputados. Recuperado de: https://www.bcn.cl/historiadelaley/nc/ historia-de-la-ley/464I/

Presidencia de la República (20I4). Instructivo presidencial no. 7. Santiago de Chile. Recuperado de:http://www.minjusticia.gob.cl/media/20 I5/05/Instructivo-Presidencial-sobreParticipaci\%C3\%B3n-Ciudadana.pdf

Presidencia de la República (2016). Decreto 00 I. Crea el Consejo Nacional de Participación Ciudadana y Fortalecimiento de la Sociedad Civil. Recuperado de: https://www.leychile. $\mathrm{cl} /$ Navegar?idNorma $=1089186$

Ramió, C., y Salvador, M. (2005). Instituciones y nueva gestión pública en América Latina. Barcelona: Fundación Cidob.

Wilson, W. (1887). "The Study of Public Administration". Political Science Quarterly, 2(2), 197-222. 